\title{
Ensaio
}

Lisiane Seguti Ferreira ${ }^{1,6}$

Dario Pallhares ${ }^{2}$

Vladimir Ferreira Seguti ${ }^{3,6}$

Marília Gava ${ }^{4,6}$

Antônio Carlos Estima Marasciulo ${ }^{5,6}$

\section{Epilepsia e Previdência Social: a decisão médico-pericial}

\author{
Epilepsy and Social Security: the medical decision making on \\ disability compensation
}

${ }^{1}$ Médica Neurologista, Professora Adjunta da Universidade de Brasília (UNB), Brasília, DF, Brasil.

${ }^{2}$ Médico do Trabalho do Hospital das Forças Armadas, Brasília, DF, Brasil.

${ }^{3}$ Médico Ortopedista do Hospital das Forças Armadas, Brasília, DF, Brasil.

${ }^{4}$ Médica. Consultora técnica do Instituto Nacional do Seguro Social (INSS) responsável pela elaboração de procedimentos e protocolos para subsidiar a avaliação médico pericial. Brasília, DF, Brasil.

${ }^{5}$ Médico Epidemiologista do Hospital Universitário da Universidade Federal de Santa Catarina (UFSC), Florianópolis, SC, Brasil.

${ }^{6}$ Médico Perito do Instituto Nacional do Seguro Social (INSS).

Trabalho não subvencionado e sem conflitos de interesse.

Contato:

Dario Palhares

E-mail:

dariompm@unb.br

\section{Resumo}

A maior dificuldade encontrada no manejo de pacientes com epilepsia frente aos seguros saúde é que, apesar de todo o aparato tecnológico de que se dispõe atualmente, o diagnóstico continua sendo predominantemente clínico, não havendo critérios objetivos na definição da incapacidade laborativa. Este ensaio tem como propósito discutir necessidades e parâmetros e apresentar uma proposta de aperfeiçoamento de relatório a ser preenchido pelo médico assistente a fim de traçar estratégias para que o perito médico defina, com maior segurança, a incapacidade laborativa em segurados portadores de epilepsia. A proposta discute aspectos relacionados a diagnóstico, tratamento e prognóstico, assim como fatores que interferem na capacidade de trabalho, visando auxiliar a decisão médico-pericial acerca da concessão ou não de benefícios.

Palavras-chave: laudo técnico pericial; incapacidade laborativa; seguro-saúde; epilepsia.

\begin{abstract}
Regarding health insurances, the major difficulty in handling patients with epilepsy is that the diagnosis is basically clinical, and there is not a totally objective criteria to define work incapacity, even with all the existing modern technology. In this essay we discuss needs and parameters, propose guidelines for the report filled up by assistant physicians, and formulate strategies to help medical experts defining work incapacity for workers with epilepsy, with a greater margin of accuracy. We discuss aspects related to diagnosis, treatment, prognosis, and list facts that can eventually interfere in the ability to work, contributing for medical experts to decide whether they should or not grant disability benefits.
\end{abstract}

Keywords: expert assessment; work incapacity; health insurance; epilepsy. 


\section{Introdução}

A epilepsia é uma condição médica comum que afeta entre $1 \%$ e $2 \%$ da população. É uma questão de saúde pública e não tem barreiras sociais, étnicas, geográficas, etárias ou sexuais. No Brasil, segundo estimativas do Ministério da Saúde (Datasus), surgem pelo menos 150 mil casos novos ao ano (incidência de 100/100.000) e 1,5 a 2,5 milhões de casos com epilepsia ativa (prevalência de 1-1,5\%), sendo que $25 \%$ destes apresentam prognóstico reservado que se agrava frente à baixa acessibilidade aos métodos de diagnóstico e tratamento (GOMES, 2000).

Constituindo-se em uma das principais causas neurológicas de afastamento do trabalho com impacto social e econômico negativos na sociedade, a epilepsia atinge adultos na fase produtiva, interfirindo em sua capacidade de trabalho. Há menos oportunidades de emprego decorrentes das limitações e do estigma associado à doença (TREVISOL-BITTENCOURT et al., 2001; SALGADO; SOUZA, 2002; PATO-PATO et al., 2004).

O indivíduo com epilepsia é frequentemente alijado do mercado do trabalho, o que também traz impactos negativos para o próprio trabalhador, visto que o trabalho é um dos aspectos mais importantes para a autoestima e a realização pessoal (PALHARES; SANTOS, 2012). Além disso, a permanência do indivíduo no trabalho em geral é curta e os índices de desemprego são elevados. Tais fatores podem estimular o trabalhador a procurar, precocemente, os benefícios de seguradoras de saúde, como o Instituto Nacional de Seguro Social (INSS).

A incapacidade laborativa é definida como a impossibilidade do desempenho das funções específicas de uma atividade (ou ocupação) em consequência de alterações morfopsicofisiológicas provocadas por doença ou acidente (TAVARES, 2009). A maior dificuldade encontrada na avaliação da incapacidade laborativa do segurado portador de epilepsia é a ausência de critérios objetivos para a definição dessa incapacidade e a dependência frequente da informação do segurado e dos seus familiares (TELLA; FONSECA; BERTUQUI, 1996; GOMES, 2009; COCKEREL; SHORVON, 1997; FERREIRA et al., no prelo*).

A falta de critérios objetivos, especificamente para epilepsia, pode dificultar a decisão do perito. Neste contexto, e em face do caráter crônico da doença, negar o benefício ao segurado com epilepsia, incapaz para o trabalho, pode implicar em danos irreversíveis dos pontos de vista pessoal e social. Por outro lado, a concessão equivocada de longos períodos de afastamento pode ser iatrogênica e comprometer, definiti- vamente, as chances de integração social e realização pessoal deste indivíduo (SALGADO; SOUZA, 2002).

O perito do INSS embasa a decisão médico-pericial praticamente nos dados do relatório médico trazido pelo segurado, o qual, conforme levantamento anterior, referente aos anos de 2003 a 2008 realizado em Brasília, Distrito Federal (FERREIRA et al., no prelo*), é bastante incompleto, com falhas na caracterização da história clínica, do diagnóstico e do tratamento.

Assim, considerando que a quase totalidade das perícias do INSS é feita por peritos não neurologistas, é necessário que haja diretrizes claras e objetivas que permitam nortear a conduta mais adequada frente aos casos de epilepsia. Neste sentido, uma equipe de peritos especialistas da área de Clínica Médica desenvolveu um material de cunho técnico-médico que discorre sobre conceitos, critérios diagnósticos, tratamentos, prognósticos e faz sugestões quanto ao período ideal de afastamento para diversas condições médicas, analisadas sob a ótica da medicina baseada em evidências (INSTITUTO NACIONAL DO SEGURO SOCIAL, 2009). Diferentemente dessas diretrizes, o enfoque deste ensaio consiste no aperfeiçoamento do laudo elaborado pelo médico assistente que atende o paciente com epilepsia.

Dentro do paradigma desenvolvido pelo programa "Epilepsia Saindo das Sombras" (YACUBIAN, 2000), uma iniciativa mundial que pretende desmistificar aspectos relacionados à doença e ao doente com epilepsia e estabelecer um modelo de atendimento integral a estas pessoas, o presente texto tem por objetivos:

a) propor um modelo de relatório que contenha informações necessariamente preenchidas pelo médico assistente na elaboração do seu laudo;

b) orientar o perito no delineamento do quadro clínico do segurado com epilepsia;

c) fornecer subsídios ao perito para definição de incapacidade laborativa em epilepsia;

d) auxiliar na avaliação pré-admissional de indivíduos com epilepsia.

\section{A caracterização do quadro clínico do periciando}

A epilepsia é definida como um grupo de doenças que têm em comum o paroxismo de descargas neuronais aberrantes, que recorrem na ausência de condições tóxicas, metabólicas ou febris. A crise epiléptica é uma manifestação estereotipada, em que o paciente pode apresentar sinais motores, experiên- 
cias psíquicas, distúrbios autonômicos com ou sem perda de consciência. Geralmente, tem início súbito, curta duração, cessa espontaneamente e pode ser acompanhado de sonolência e quadro confusional - período pós-ictal (COCKEREL; SHORVON, 1997).

A epilepsia é episódica e, entre as crises, o exame físico e mesmo o eletrencefalograma podem ser perfeitamente normais (SANDER; HART, 1999; COCKEREL; SHORVON, 1997; GUERREIRO et al., 2000). Esse conceito é fundamental para definir a conduta na perícia médica.

Dentro do modelo cibernético de Wiener (CONWY; SIEGELMAN; ALEXANDERSON, 2006) da sequência insumo-processamento-produto, a tomada de decisão médico-pericial é o produto final do processamento de informações clínicas. Assim, para que o produto final (no caso, a decisão do perito) seja consistente e de boa qualidade, é preciso que os insumos sejam de boa qualidade. Portanto, o primeiro passo para a decisão consciente e segura do perito é a obtenção de um insumo de boa qualidade, ou seja, a ênfase na correta caracterização do quadro clínico e do diagnóstico visando embasar a decisão final.

Até a decisão médico-pericial, que determinará o direito de afastamento do trabalho por motivo de doença e a concessão ou não do benefício previdenciário, o trabalhador enfrentará algumas etapas. Todo trabalhador submetido ao regime da Previdência Social deverá contribuir, obrigatoriamente, por pelo menos 12 meses para alcançar a condição de segurado, especialmente no caso de doenças como epilepsia, que não isenta carência (BRASIL, 1991).

Caso o segurado necessite se afastar do trabalho por mais de 15 dias, inicia-se o processo de solicitação de auxílio doença junto ao INSS. De posse de toda documentação necessária, que deve comprovar não apenas a qualidade de segurado, mas também a data de início da doença e da incapacidade, o trabalhador será submetido à avaliação médico-pericial (BRASIL, 1991).

Nesta avaliação, os dados clínicos e de exames complementares serão analisados pelo perito, juntamente com o exame neurológico, para uma decisão judiciosa quanto à existência ou não de incapacidade. O perito poderá decidir que há incapacidade por um prazo definido (Data da cessação do benefício - DCB); que se trata de incapacidade por doença ou lesão de evolução prolongada e incerta, devendo ser reexaminado após um prazo de dois anos (Revisão em 2 anos - R2); que há incapacidade definitiva para a atividade usual, sendo encaminhado para a reabilitação profissional (Reabilitação profissional - RP); ou que o segurado preenche critérios para incapacidade definitiva omniprofissional (Limite indefinido - aposentadoria por invalidez - LI) (BRASIL, 1991).

\section{Detalhamento do quadro clínico-laboral}

Especificamente no que tange à caracterização da epilepsia, há dois grandes grupos de perguntas básicas que devem constar da avaliação do médico assistente e que servirão de base para que o perito possa definir incapacidade: perguntas relacionadas ao diagnóstico e perguntas relacionadas ao histórico do tratamento (sendo que os dados obtidos em ambos os grupos reforçam-se uns aos outros).

Perguntas relacionadas ao diagnóstico:

a) Quais os tipos de crises?

b) Dado fundamental: qual a frequência aproximada das crises? Semanais? Mensais? Esporádicas? Quantas crises houve no mês passado? E nos últimos três meses?

c) A epilepsia é idiopática? Ocorre aura?

d) Tem antecedente de trauma neonatal, trauma cranioencefálico, tumor cerebral, acidente vascular encefálico, ingestão de substâncias tóxicas, distúrbio metabólico?

e) Há história familiar de epilepsia?

f) Há documentação que comprove que o segurado/requerente tem epilepsia crônica?

g) O relato de crises é confirmado pelo acompanhante? Há antecedente de crises psicogênicas não epilépticas?

h) Existe antecedente de estado de mal epiléptico?

i) Foi submetido a estudo com monitoração vídeo-eletrencefalográfica?

A devida caracterização da epilepsia, uma condição cujo diagnóstico é feito principalmente com bases clínicas, deve ser feita junto ao paciente e conferida/confrontada com os dados contidos no relatório do médico assistente.

É importante também que o perito esteja atento para o fato de que as crises epilépticas geralmente têm curta duração e não superam dois a três minutos. A ocorrência de crises muito elaboradas, muito longas e/ou bizarras e que ocorram coincidentemente no consultório do perito deve alertar para a ocorrência de distúrbios não epilépticos.

Exceção aplica-se às crises parciais do lobo frontal, muitas vezes interpretadas como psicogênicas pelo seu caráter bizarro. Nesta circunstância, é recomendável que o perito solicite um parecer do médico assistente (deixar pendente por sistema de informação do médico assistente - SIMA) quanto à possibilidade de epilepsia frontal e/ou estado de mal não epiléptico. Deste modo, a perícia só será concluída depois que o segurado trouxer os documentos médicos (que 
incluem laudos, exames complementares, dados de exame neurológico) que comprovem ou não a incapacidade referida. Quando as informações contidas no relatório do médico assistente forem esclarecedoras e completas e subsidiarem a decisão médico-pericial, é dispensável o preenchimento do SIMA.

Perguntas relacionadas ao tratamento:

a) Foi identificada a etiologia?

b) Está usando fármacos antiepilépticos (FAEs)? Quais os FAEs utilizados?

c) Qual o histórico de uso dos FAEs: sempre usou esse esquema? Houve associação a novas drogas? Houve substituição? Desmame de alguma droga? Há registro dos níveis séricos das drogas antiepilépticas?

d) Passa por avaliações periódicas com o neurologista ou então com o clínico geral?

e) É candidato à cirurgia de epilepsia?

Todas estas informações devem estar contidas no relatório do médico assistente. $\mathrm{O}$ perito, então, de posse dos relatórios e de outros documentos comprobatórios, realiza a perícia, verificando a exatidão dos dados apresentados e, quando possível, registrando a medicação em uso. As drogas antiepilépticas mais comuns, seus efeitos colaterais e a dose mínima eficaz estão disponibilizadas no Quadro 1. É importante que o perito procure por sinais de intoxicação, os quais também podem fornecer pistas quanto à adesão terapêutica e a gravidade do quadro clínico.

Um aspecto limitado ao universo do periciando é a adesão correta ao esquema posológico dos FAEs (MARTIN et al., 2005). O perito pode solicitar a dosagem sérica do medicamento em uso, caso suspeite de baixa adesão do periciando ao tratamento, e o ideal seria que a coleta sanguínea pudesse ser feita já ao momento do exame pericial. Esta dosagem, no entanto, fornece apenas uma visão pontual e parcial do nível sérico, que não necessariamente reflete o tratamento. A real dimensão destes dados só poderá ser fornecida pelo médico, de preferência neurologista, que acompanha o doente.

Sinais indiretos de gravidade devem ser sempre documentados: presença ou ausência de sinais de intoxicação ao exame físico (Quadro 1); uso de politerapia em doses elevadas; internações frequentes; ocorrência de estado de mal epiléptico; participação em programa de seleção para tratamento cirúrgico; sinais de deterioração psíquica e/ou neurológica; comorbidade psiquiátrica grave.

Quadro 1 Principais drogas antiepilépticas, dose e efeitos colaterais

\begin{tabular}{|c|c|c|}
\hline Fármacos antiepilépticos & Dose terapêutica média & Principais efeitos colaterais \\
\hline Fenobarbital & $100-200 \mathrm{mg} / \mathrm{dia}$ & $\begin{array}{l}\text { Fadiga, sedação, depressão, dificuldade de } \\
\text { concentração, rash, contratura de Dupuytren, } \\
\text { distúrbios hematológicos }\end{array}$ \\
\hline Fenitoína & $300-500 \mathrm{mg} / \mathrm{dia}$ & $\begin{array}{l}\text { Mudanças cognitivas e comportamentais, } \\
\text { perda da coordenação e equilíbrio, distúrbios } \\
\text { gastrintestinais e alterações na pele }\end{array}$ \\
\hline Carbamazepina & $600-800 \mathrm{mg} / \mathrm{dia}$ & $\begin{array}{l}\text { Sonolência, fadiga, tontura, visão turva, rash } \\
\text { cutâneo, diplopia, ataxia, leucopenia, elevação } \\
\text { de enzimas hepáticas, retensão hídrica }\end{array}$ \\
\hline Valproato & $500-3.000 \mathrm{mg} / \mathrm{dia}$ & $\begin{array}{l}\text { Anorexia, náuseas e vômitos, ganho excessivo } \\
\text { de peso, elevação de enzimas hepáticas, queda } \\
\text { de cabelo, distúrbios endócrinos }\end{array}$ \\
\hline Benzodiazepínicos & $\begin{array}{l}\text { Variável. Geralmente não é } \\
\text { utilizado em monoterapia }\end{array}$ & $\begin{array}{l}\text { Fadiga, sonolência, ataxia, distúrbio de compor- } \\
\text { tamento, visão borrada, diplopia e hipotonia }\end{array}$ \\
\hline
\end{tabular}

Fonte: Adaptado de Yacubian (2004). 
Um roteiro que pode ser utilizado para preenchimento do relatório do médico assistente é disponibilizado a seguir:

1) O paciente tem epilepsia? - data de início

2) Qual a causa da epilepsia?

3) Qual a frequência das crises por mês, aproximadamente?

4) Qual o tipo de crise?

5) Há algum dado de exame complementar relevante?

6) Quais os tratamentos, doses anteriores e efeitos colaterais do tratamento atual?

7) É candidato à cirurgia de epilepsia?

8) Há suspeita de crises psicogênicas não epilépticas?

9) Há suspeita de ganho secundário?

10) Faz tratamento psiquiátrico?

11) Proposta de tempo de afastamento e justificativa.

O ideal é que os relatórios analisados pelo perito médico contenham minimamente estas informações. Assim, o perito analisa o relatório, registra o tratamento, procura por sinais de intoxicação medicamentosa e pode definir com maior segurança se há ou não incapacidade laborativa.

Abaixo, segue um exemplo fictício de um laudo elaborado por médico assistente:

Paciente, 30 anos, apresenta epilepsia desde os 23 anos, do tipo epilepsia do lobo temporal, secundária à atrofia hipocampal direita. Mantém média de duas crises mensais, a despeito da adesão medicamentosa. Apresenta crises focais, atualmente, mas já apresentou crises tônico clônicas generalizadas. Já fez tratamento com fenobarbital (dose de $200 \mathrm{mg} /$ dia) com sinais de intoxicação, fenitoína na máxima dose tolerada. Atualmente, utiliza carbamazepina na dose de $1 \mathrm{~g} /$ dia, sem efeitos colaterais evidentes, mas com controle parcial das crises (dose em progressão). No momento, ainda não é candidato à cirurgia de epilepsia. Não há suspeita de pseudocrises e não há comorbidade psiquiátrica. Para a profissão atual (assistente administrativo) não há necessidade de readaptação ou reabilitação profissional. Como apresenta somente crises focais e está parcialmente controlado (duas crises/mês), não há impedimento para o exercício da profissão.

\section{A decisão do perito médico}

As informações assim coletadas serão processadas, e as decisões finais possíveis são as seguintes:

a) o periciando é plenamente apto ao trabalho. b) o periciando deve se afastar do trabalho por tempo determinado.

c) o periciando deve se afastar do trabalho por tempo indeterminado.

d) o periciando deve ser reabilitado/remanejado para outra função.

e) o periciando está total e permanentemente incapaz para o trabalho.

A decisão do perito deve considerar que, mesmo com todo o arsenal terapêutico existente, cerca de $20 \%$ dos pacientes continuarão apresentando crises epilépticas. Desses 20\%, somente 5\% apresentarão epilepsia grave, tornando-se incapazes para o trabalho e dependentes de supervisão externa contínua (SHORVON; REYNOLDS, 1982). Uma pequena minoria apresentará deterioração física e intelectual progressiva (SANDER; HART, 1999; JALLON; 2004).

Os principais fatores que prejudicam a capacidade laboral são: a frequência das crises, a ocorrência de efeitos colaterais das drogas antiepilépticas e a presença de outras comorbidades (CUKIERT, 2006).

Existem situações bastante típicas e bem delimitadas, em que é possível a tomada de condutas mais precisas, mas também há situações complexas que dão margem à discussão e à subjetividade dentro do próprio contexto clínico da epilepsia e da perícia médica (GOMES, 2009). De todo modo, será muito difícil e imprecisa qualquer generalização sobre uma condição tão abrangente como a da epilepsia, que se refere a um conjunto de síndromes com diversas etiologias, evoluções, tratamentos e prognósticos (GUERREIRO et al. 2000).

No contexto clínico, a anamnese é soberana. Contudo, esse axioma nem sempre é verdadeiro no âmbito médico-pericial pela própria natureza das relações humanas. No contexto clínico, a informação e a confiança do paciente no médico e vice-versa é essencial para o sucesso do tratamento; no contexto pericial, a verdade dos fatos pode significar a perda ou a não concessão de algum benefício pecuniário, ou seja, informar ao perito que houve total controle de crises pode implicar na suspensão do benefício e o imediato retorno ao trabalho, o que muitas vezes não representa a vontade do trabalhador (SHAFER, 1988). Assim, além da informação prestada pelo segurado, o relato de testemunhas ajuda também no diagnóstico diferencial da epilepsia.

No que tange ao contexto pericial, é importante avaliar quanto ao periciando:

a) Se o quadro clínico é, de fato, epilepsia, se há comorbidades psiquiátricas e se outros diagnósticos diferenciais foram adequadamente afastados. O Quadro 2 sintetiza os 
principais diagnósticos diferenciais a serem considerados.

b) Se a epilepsia é de início recente ou se o indivíduo apresenta uma condição crônica, prévia à condição de trabalhador e/ou à solicitação do benefício.

c) Se o tratamento é efetivo, se há indícios de má adesão terapêutica.

d) Se a epilepsia configura-se como refratária e se há sinais de intoxicação medicamentosa.

Além disso, uma vez que a epilepsia apresenta restrições a certos tipos de ocupação, que representem riscos não somente para si, mas também a terceiros, o perito deve considerar as informações que apontem necessidade de reabilitação/readequação profissional:

a) Trabalha em ambiente que não restrinja sua condição clínica e permita o tratamento?

e) Há fatores que afetam diretamente sua capacidade de recuperação?

f) Apresentou estado de mal epiléptico ou lesões graves enquanto trabalhava?

g) Igualmente, as condições e a natureza do ambiente de trabalho devem ser levadas em conta para a determinação da duração do afastamento do trabalho. Além disso, tarefas que expõem o indivíduo e outros a riscos não devem ser realizadas por aqueles com epilepsia (TELLA; FONSECA; BERTUQUI, 1996; COCKEREL; SHORVON, 1997).

\section{Quadro 2 Diagnósticos diferenciais}

\begin{tabular}{|c|c|}
\hline Diagnósticos diferenciais & Descrição de principais achados \\
\hline Síncope & $\begin{array}{l}\text { Sensação de flutuação, embaçamento visual, distúrbios autonômicos que melhoram } \\
\text { com a posição horizontal }\end{array}$ \\
\hline Episódios isquêmicos transitórios & $\begin{array}{l}\text { Dominam os sintomas negativos como fraqueza e perda de sensibilidade, ao invés } \\
\text { das parestesias observadas nas crises somatossensitivas ou os abalos vistos nas } \\
\text { crises motoras }\end{array}$ \\
\hline Tiques & $\begin{array}{l}\text { Distúrbio de movimento que é suprimido pela vontade e não tem a natureza } \\
\text { episódica das crises parciais simples }\end{array}$ \\
\hline Hiperventilação & Caracteriza-se por disestesias periorais nos pés e nas mãos, geralmente bilaterais \\
\hline Espasmo hemifacial & Fenômeno contínuo, recorrente que se agrava com estado emocional \\
\hline Distúrbios do sono & $\begin{array}{l}\text { Incluem terror noturno, sonambulismo, enurese, apneia do sono, bruxismo, jactatio } \\
\text { captis e movimentos periódicos do sono. Geralmente, estes episódios têm maior } \\
\text { duração e podem ser interrompidos pelo despertar }\end{array}$ \\
\hline Crises não epilépticas & $\begin{array}{l}\text { Confundidas principalmente com crises parciais complexas. Podem ser comprovadas } \\
\text { por uma responsividade parcial, amnésia parcial ou resposta a estímulos dolorosos }\end{array}$ \\
\hline Narcolepsia & $\begin{array}{l}\text { Diagnóstico principalmente com a cataplexia, que compõe o quadro de narcolepsia, } \\
\text { juntamente com alucinações hipnagógicas e paralisia do sono. A característica prin- } \\
\text { cipal é a precitação pela emoção, tal como o riso, cursando com preservação } \\
\text { da consciência }\end{array}$ \\
\hline Desordens metabólicas & $\begin{array}{l}\text { Distúrbio hidroeletrolítico que em geral pode causar eventos episódicos transitóri- } \\
\text { os. Na hipoglicemia, o paciente pode queixar-se de sudorese, fome e os episódios } \\
\text { ocorrem principalmente após jejum prolongado. }\end{array}$ \\
\hline Amnésia global transitória & $\begin{array}{l}\text { Ocorre em idosos e em indivíduos de meia-idade como episódios únicos de amnésia } \\
\text { completa por um período de várias horas. A despeito da preservação da consciência, } \\
\text { o paciente pergunta, repetidas vezes, questões relacionadas a tempo e lugar }\end{array}$ \\
\hline Enxaqueca & $\begin{array}{l}\text { Fenômeno paroxístico que pode produzir sintomas sensoriais. Geralmente, início } \\
\text { gradual tem a característica fundamental é a cefaleia e a duração é prolongada. }\end{array}$ \\
\hline
\end{tabular}

Fonte: Adaptado de Cockerel; Shorvon, 1997. 
O Quadro 3 lista determinadas situações com as quais o perito se depara. Na primeira, há crises que não se enquadram no conceito de epilepsia, com padrão de crises bizarras, não sugestivas de epilepsia frontal (conforme relatório do neurologista), ocorrendo coincidentemente dentro das dependências onde será feita a perícia. A incapacidade laborativa, nestes casos, por CID G40 (Epilepsia) e similares, é pouco provável.

Outra situação é a do segurado com epilepsia recém-diagnosticada, candidato a uma vaga de emprego. Tais segurados, no exame admissional, são aptos para a maioria das profissões, exceto aquelas nas quais uma crise epiléptica colocaria em risco a sua integridade e/ou a de terceiros: operação de máquinas pesadas, direção de veículos, porte de armas etc. No que tange à maioria das ocupações (construção civil [atividades em solo], artesanato, pesca etc.), o paciente com epilepsia é, no geral, apto ao trabalho (SARMENTO; MINAYO-GOMEZ, 2000).

Segurados com diagnóstico recente de epilepsia fazem jus a um período de afastamento de 30 dias, prorrogáveis por mais 30 , que é um período razoável para a conclusão de exames complementares e para a fase de adequação terapêutica. Segurados controla- dos, com epilepsia de longa data, podem fazer jus ao benefício por curto período, durante a fase de descompensação e reajuste de FAEs. A recorrência de um episódio isolado nestes casos, por si só, não configura nem justifica afastamento ao trabalho (INSTITUTO NACIONAL DO SEGURO SOCIAL, 2009)

Nos casos de epilepsia refratária, o segurado pode ter direito não apenas a longos períodos de afastamento, mas também à indicação de afastamento definitivo. No entanto, o aspecto mais difícil para o perito é a confirmação de que realmente se trata de epilepsia refratária e não de casos de má adesão terapêutica, de diagnóstico equivocado e de ocorrência de crises de natureza não epiléptica. Particularmente neste ponto, é imprescindível a elaboração de um relatório bem fundamentado pelo médico assistente para subsidiar a decisão do perito.

O conceito de "epilepsia refratária” tem sido reformulado (JANSZKY et al., 2010). A Liga Internacional contra a Epilepsia (International League Against Epilepsy - ILAE) propôs que a resistência ao tratamento seja definida como a falha em alcançar o controle de crises utilizando-se duas FAEs com boa tolerabilidade, apropriadamente escolhidas, em mono ou politerapia (KWAN et al., 2010).

Quadro 3 Conduta médico-pericial e respectivas condições em epilepsia

\begin{tabular}{|c|c|}
\hline Conduta pericial & Situações em epilepsia \\
\hline $\begin{array}{l}\text { Incapacidade deve ser forte- } \\
\text { mente questionada }\end{array}$ & $\begin{array}{l}\text { - Crises bizarras, prolongadas e polimórficas, com manifestações clínicas que não se } \\
\text { enquadram nas síndromes epilépticas e que ocorram no momento exato da perícia. } \\
\text { - Subdose de medicamentos no contexto de epilepsia acompanhada como refratária. } \\
\text { - Crises exclusivamente noturnas para o trabalhador com carga de trabalho habitual diurna. }\end{array}$ \\
\hline Data para cessação do benefício & $\begin{array}{l}\text { - Epilepsia recém diagnosticada em fase de investigação e adaptação do uso de drogas } \\
\text { antiepilépticas: concessão por } 30 \text { dias e, se ainda instável, prorrogável até } 60 \text { dias. }\end{array}$ \\
\hline Reabilitação profissional & $\begin{array}{l}\text { - Epilepsia bem definida no contexto de profissões de risco (motorista, operador de } \\
\text { máquinas pesadas, mergulho etc). }\end{array}$ \\
\hline Revisão em dois anos (R2) & $\begin{array}{l}\text { - Epilepsia refratária, bem documentada, em politerapia com doses elevadas. É desejável } \\
\text { anotar sinais clínicos de intoxicação e a realização de dosagem sérica das drogas } \\
\text { prescritas. } \\
\text { - Casos elegíveis para cirurgia. }\end{array}$ \\
\hline Limite indefinido (LI) & $\begin{array}{l}\text { - Epilepsia refratária de longa data, em acompanhamento em Centros Terciários, associ- } \\
\text { ada à comorbidade psiquiátrica grave e/ou eletroencefalograma (EEG) persistentemente } \\
\text { alterado, e /ou má resposta cirúrgica. }\end{array}$ \\
\hline
\end{tabular}

Fonte: Adaptado de Instituto Nacional do Seguro Social (2009). 
Para fins do contexto pericial, uma epilepsia é refratária se isso implica em incapacidade laboral persistente e, para fins de decisão médico-pericial, pode ser considerada, com as devidas adaptações, se o periciando se enquadrar em um ou mais casos:

a) Tratamento com pelo menos dois FAEs, na máxima dose tolerada, adequados ao tipo de epilepsia, separadamente ou associados, por um período de tempo e com ótima aderência terapêutica;

b) Realização de monitoração vídeo-eletrencefalográfica, com resultados anormais, pois é um exame tipicamente indicado para casos de refratariedade;

c) Indicação neurocirúrgica por parte da equipe clínica.

Em caso de segurados afastados por mais de seis meses, é recomendável a solicitação de cópia do prontuário médico ou SIMA. Pelo próprio curso da doença, nas grandes cidades, pacientes com epilepsia grave, que exige longo tempo de afastamento, costumam ser acompanhados em hospitais de referência. Pode não ser essa a realidade em boa parte do Brasil, mas o perito deve colocar ao menos em suspeição se nas cidades com mais recursos um periciando com história de epilepsia refratária não estiver sendo acompanhado por especialistas em unidades mais complexas.

Se o segurado for candidato cirúrgico, a concessão de benefício com revisão em dois anos (R2) é uma alternativa. Caso ele retorne com persistência de crises e comorbidades (distúrbios psiquiátricos), a indicação de aposentadoria por invalidez pode ser a única opção. Segurados com idade superior a 50 anos e diagnóstico bem definido de epilepsia refratária têm indicação de aposentadoria.

Entre o periciando que não faz jus à concessão de benefícios e o segurado com epilepsia refratária, há um contingente significativo de trabalhadores que usam uma ou duas drogas antiepilépticas, mas que apresentam crises esporádicas e que, na maioria dos casos, não incapacitam para o trabalho. Para tais situações, persiste uma margem de subjetividade, tanto na decisão do perito, como também para o neurologista que acompanha o paciente (GOMES, 2009).

\section{Considerações finais}

A decisão médico-pericial quanto à incapacidade para o trabalho esbarra em uma série de dificuldades, não apenas pela baixa confiabilidade das informações fornecidas pelo segurado, mas também pela falta de um marcador para confirmação de epilepsia ativa. A proposta dos autores discute aspectos relacionados ao diagnóstico, ao tratamento e ao prognóstico e a situações bem delimitadas para a concessão e para a não concessão de benefícios. Os periciandos que se situarem entre esses dois extremos deverão ser avaliados caso a caso.

As considerações aqui apresentadas não se aplicam à epilepsia secundária a tumores, traumatismo cranioencefálico, etilismo etc., cuja análise deve levar em conta a doença de base.

É preciso reafirmar que não há espaço para qualquer generalização em epilepsia e que a definição de incapacidade deve levar em conta não apenas a natureza e o quadro clínico das crises, mas também o tipo de ocupação, a escolaridade e as possibilidades de reinserção no mercado de trabalho.

Por fim, face à complexidade de ambos os temas, incapacidade e epilepsia, os autores reconhecem que este trabalho é preliminar e que o mais importante é aprofundar no guia de informações do médico assistente e em meios sistemáticos de analisar as informações prestadas por ele.

\section{Contribuição de autoria}

Todos os autores foram responsáveis pela elaboração do projeto, pela revisão bibliográfica, pela análise dos fatos e todos estão de acordo com esta versão final do texto.

\section{Referências}

BRASIL. Lei 8.213, de 24 de Julho de 1991. Dispõe sobre os planos de benefício da previdência social e dá outras providências. Diário Oficial [da] República Federativa do Brasil, Brasília, DF, 25 jul. 1991. Disponível em: <http://www.planalto.gov.br/ccivil_03/ leis/L8213cons.htm > . Acesso em: 12 nov. 2012.
COCKEREL, C. O.; SHORVON, S. D. Epilepsia: conceitos atuais. São Paulo: Lemos Editorial \& Gráficos, 1997.

CONWY, F; SIEGELMAN J.; ALEXANDERSON, G. Dark Hero of the information age: in search of Norbert Wiener, the father of Cybernetcs. The Mathematical Intelligencer, v. 28, n. 2, p. 70-73, 2006. 
CUKIERT, A. Epilepsias generalizadas. São Paulo: Segmento Farma, 2006.

GOMES, M. M. Epilepsia e incapacidade laborativa. Journal of Epilepsy and Clinical Neurophysiology, v. 15 n. 3, p. 130-134, 2009.

GOMES, M. M. Epidemiologia: distribuição, fatores de risco e considerações prognósticas. In: GUERREIRO, C. A. M. et al. Epilepsia. 2. ed. São Paulo: Lemos Editorial \& Gráficos, 2000. p. 11-21.

GUERREIRO, C. A. M. et al. Epilepsia. 3. ed. São Paulo: Lemos Editorial \& Gráficos, 2000.

INSTITUTO NACIONAL DO SEGURO SOCIAL. Diretrizes de apoio à decisão médico-pericial - Parte I. Versão preliminar para consulta pública. Brasília: Ministério da Previdência Social, 2009. Disponível em: <http://www.previdenciasocial.gov.br/arquivos/ office/4 091021-153135-494.pdf > . Acesso em: abril de 2012 .

JALLON, P. Epidemiology of drug-resistant epilepsies. Revue Neurologique, Paris, v. 160, Supl. 1, p. 22-30, jun. 2004.

JANSZKY, J. et al. Epilepsy drugs, antiepileptic drug trials and the role of evidence. Epilepsia, v. 51, n. 6, p. 1004-1009, 2010.

KWAN, P. et al. Definition of drug resistant epilepsy: consensus proposal by the ad hoc task force of the ILAE commission on therapeutic strategies. Epilepsia, v. 51, n. 6, p. 1069-1077, 2010.

MARTIN, R. et al. What are the concerns of older adults living with epilepsy? Epilepsy and Behavior, v. 7, n. 2, p. 297-300, 2005.

PALHARES, D.; SANTOS, I. L. Pre-employment medical testing in Brazil: ethical challenges. Indian Journal of Medical Ethics, v. 9, n. 4, p. 266-268, oct.dec. 2012
PATO-PATO, A. et al. The economic impact of epilepsy. Revista de Neurología, Barcelona, v. 39, n. 5, p. 450-453, 2004.

SANDER, J. W.; HART Y. M. Epilepsia: um guia prático. São Paulo: Merit Publishing International, 1999.

SALGADO, P. C. B; SOUZA, E. A. P. Impacto da epilepsia no trabalho. Avaliação da qualidade de vida. Arquivos de Neuro-Psiquiatria. São Paulo, v. 60, n. 2B, p. 442-445, junho 2002.

SARMENTO, M. R. S.; MINAYO-GOMEZ, C. A epilepsia, o epiléptico e o trabalho: relações conflitantes. Cadernos de Saúde Pública, Rio de Janeiro, v. 16, n. 1, p. 183-193, jan-mar 2000.

SHAFER, S. Q. et al. EEG and others predictors of epilepsy remission: a community study. Epilepsia, v. 29, n. 5, p. 590-600, 1988.

SHORVON, S. D.; REYNOLDS, E. H. Early prognosis of epilepsy. British Medical Journal, v. 285, n. 6356, p. 1699-1701, 1982.

TAVARES, M. L. Direito previdenciário. 11. ed. São Paulo: Impetus, 2009.

TELLA, L. M. G.; FONSECA, L. C.; BERTUQUI, C. M. Epilepsia e trabalho. Revista de Ciências Médicas, Campinas, v. 5, n. 3, p. 113-118, 1996.

TREVISOL-BITTENCOURT, P. C. et al. Condições neurológicas mais freqüentes em um ambulatório de perícia neurológica. Arquivos de Neuropsiquiatria, São Paulo, v. 59, n. 2-A, p. 214-218, 2001.

YACUBIAN, E. M. T. Tratamento medicamentoso das epilepsias. 2. ed. São Paulo: Lemos Editorial \& Gráficos, 2004.

. Epilepsia da antiguidade ao segundo milênio: Saindo das sombras. São Paulo: Lemos Editorial \& Gráficos, 2000. 